\title{
La perception de la guerre du Golfe au Vénézuela et en Amérique du sud
}

\section{Evelyn Bravo Diaz}

\section{(2) OpenEdition}

1 Journals

\section{Édition électronique}

URL : http://journals.openedition.org/conflits/101

DOI : 10.4000/conflits.101

ISSN : $1777-5345$

Éditeur :

CCLS - Centre d'études sur les conflits lilberté et sécurité, L'Harmattan

\section{Édition imprimée}

Date de publication : 17 mai 1991

ISSN : 1157-996X

Référence électronique

Evelyn Bravo Diaz, «La perception de la guerre du Golfe au Vénézuela et en Amérique du sud »,

Cultures \& Conflits [En ligne], 02 I printemps 1991, mis en ligne le 30 décembre 2002, consulté le 30 mars 2021. URL : http://journals.openedition.org/conflits/101 ; DOI : https://doi.org/10.4000/conflits. 101

Ce document a été généré automatiquement le 30 mars 2021.

Creative Commons License 


\title{
La perception de la guerre du Golfe au Vénézuela et en Amérique du sud
}

\author{
Evelyn Bravo Diaz
}

1 A l'intérieur de l'ensemble sud-américain, le Venezuela a été le pays qui, à cause du pétrole, a eu depuis longtemps les relations les plus étroites avec le golfe arabopersique. Depuis l'entrée de l'Amérique latine dans le club de grands exportateurs de pétrole brut, cette relation s'exprime à deux niveaux : celui des sociétés transnationales pétrolières (qui ont commencé à travailler dans le Golfe au début des années 50 en y transférant des cadres et des techniciens vénézuéliens), et celui du gouvernement. Ces relations ont été fructueuses comme le montrent à la fois l'extension aux producteurs du Golfe du principe fifty-fifty qui était appliqué au Venezuela dès 1943, et la création de l'OPEP en 1960, grâce aux efforts conjoints du Saoudien Abdullah Tariki, de l'Irakien Mohammed Salman et du Vénézuélien Juan Pablo Pérez Alfonso. Depuis cette date, la politique du Venezuela - comme celle à partir de 1973 d'un autre producteur sudaméricain, l'Équateur - s'est concentrée sur l'OPEP, avec comme objectifs le contrôle du niveau de la production du pétrole et la gestion de son prix. Le Venezuela a maintenu traditionnellement des positions neutres dans les conflits entre pays membres de l'OPEP, il a conservé dans la récente guerre du Golfe la même position de neutralité, bien qu'avec quelques nuances.

L'attitude du gouvernement vénézuélien devant le conflit

2 Si la position officielle du gouvernement de Caracas est d'adopter une position de neutralité dans le conflit, le Venezuela entend cependant rester un fournisseur de pétrole sûr et fable des Etats-Unis. Aussi, dès les premiers jours de la crise, augmenta-til sa production pétrolière dans le même temps qu'il proposait une réunion urgente de l'OPEP en vue de suspendre le régime de quotas de production et remplacer sur le marché international les quantités que ne pouvaient plus fournir l'Irak et le Koweï. Ces propositions, présentées quatre jours après l'invasion du Koweït par le président Carlos Andrés Pérez au vice-président américain Dan Quayle ont reçu l'approbation du président Bush lors de la visite officielle qu'il effectua peu après au Venezuela. Caracas, 
deuxième fournisseur des Etats-Unis de brut après l'Arabie Saoudite, a ainsi augmenté sa production de pétrole de 2067000 barils à $227 ? 000$ entre août et novembre $1990^{1}$.

La décision d'augmenter la production pétrolière a été suivie par les autres producteurs latino-américains. Le Mexique (241 000 barils), l'Équateur (10 000 barils), l'Argentine (25 000). Si la Colombie, de son côté, a vu diminuer sa production, malgré la promesse du président Gaviria de l'augmenter de 28000 barils, cela s'explique par les actions de sabotage des installations pétrolières colombiennes par divers groupes de guérillas.

Pour organiser la réunion de l'OPEP qui devait décider de l'augmentation de la production, le chancelier Reinaldo Figueredo a effectué en août 1990 la tournée des pays membres, faisant $\mathrm{du}$ Venezuela le principal artisan de cette politique d'augmentation de la production, et de stabilisation des prix hors de la zone du conflit. Le plan rédigé par l'Arabie Saoudite pour cette réunion extraordinaire de l'OPEP fut rejeté par l'Algérie, l'Iran et l'Indonésie, qui considérèrent que de telles initiatives faisait le jeu des groupes de pression nord-américain.

5 En adoptant cette position, le gouvernement vénézuélien a considéré qu'il serait en mesure d'obtenir un traitement plus favorable de la part des pays industrialisés du Nord: des garanties à un accès plus grand et plus facile au marché, indépendamment de ce qui se produirait sur le marché international pétrolier, un accord pour alléger la charge de la dette externe, identique à celui obtenu par l'Egypte. Dans la presse vénézuélienne, les spéculations se multiplièrent pour évaluer qu'elles seraient les recettes supplémentaires dont bénéficierait le budget, quel serait l'usage le plus adéquat de ces revenus? Les observateurs manifestèrent également la crainte de voir l'argent escompté se volatiliser sous l'effet de la corruption.

On a été jusqu'à créer un Fond de stabilisation macro-économique pour préserver de la "voracité étatique" - sinon la totalité, du moins l'essentiel - de ces rentrées extraordinaires, estimées approximativement à 4 milliards de dollars pour le 2ème semestre de l'année 1990.

7 Le surplus de ressources que le conflit du Golfe apporta au Venezuela a finalement été utilisé à la relance du plan d'investissement 1991/1996 dans les secteurs du pétrole, de la pétrochimie, du charbon, du gaz... établis par le holding pétrolier national "Petroleos de Venezuela" (PDVSA). Ce plan, dont le montant total s'élève à 52 milliards de dollars, envisage d'augmenter le potentiel de production pétrolière de 2,9 millions à 3 millions et demi de barils par jour et d'atteindre en l'an 2000,4 millions de barils. Il a suscité un grand débat sur sa "faisabilité" et sur ses objectifs finaux en raison des perspectives d'évolution du marché pétrolier à moyen terme, marqué notamment par le nouveau plan énergétique américain que le président Bush annonce pour l'après-guerre, ainsi que par les projets européen et japonais d'économie d'énergie, de création, ou de développement, d'énergie de substitution.

8 Sur un plan régional, une réunion des chanceliers du Groupe de Rio a été organisée à Caracas le 28 janvier 1991. Cette réunion avait pour objet d'analyser l'impact de la guerre du Golfe sur l'Amérique latine. Un document de base préparé par l'OLADE (Organisation latino-américaine de l'énergie), la SELA (Système économique latinoaméricain) et la $\mathrm{CEPAL}^{2}$ (Commission économique pour l'Amérique latine et les Caraïbes) disait en substance :

- Le conflit se déclenche à une époque où l'Amérique latine et les Caraïbes sont dans 
une situation de faiblesse, après la "décennie perdue". Dans un continent en proie à une effort d'ajustement structurel, le conflit ajoute un nouveau facteur d'incertitude.

10

- L'ensemble régional - qui produit 6,5 millions de barils par jour, en consomme 4,4 millions, en exporte 3,9 millions et en importe 1,5 million - sera affecté par la hausse de la facture pétrolière : 21 pays latino-américains sont des importateurs nets, parmi eux Brésil et Uruguay importent respectivement 72 et $42 \%$ de leur consommation. Cette charge supplémentaire est très coûteuse pour les économies les plus faibles, comme celles des États de l'Amérique Centrale. Quant aux pays exportateurs, s'ils tirent un bénéfice économique immédiat de la hausse des prix, les fluctuations importantes des cours du pétrole font néanmoins peser sur leurs économies des risques à moyen et à long terme certains.

11

- Les effets économiques seront donc: aggravation des tendances inflationnistes, impact sur la balance des paiements, augmentation du prix des importations, effet sur les finances publiques et les programmes $\mathrm{d}$ ajustements structurels.

Pour faire face à ces effets, il est prévu :

- Des changements de structure dans la politique énergétique de la région pour favoriser une plus grande indépendance à l'égard de la conjoncture internationale.

14

- La création d'un fond financier avec les ressources provenant des prix des matières premières.

15

- L'établissement d'une stratégie de coopération intra-régionale comprenant le secteur énergétique en vue d'atténuer les effets négatifs des fluctuations internationales des prix du pétrole.

16

- L'amélioration de l'utilisation de l'infrastructure hydraulique et charbonnière pour diminuer les coûts de production.

17

- L'augmentation de la sécurité de l'approvisionnement énergétique et l'intensification des activités d'exploration et de développement du pétrole.

Une autre mesure économique adoptée par les différents pays latino-américains fut la création de fonds nationaux afin de diminuer les effets des oscillations des prix du pétrole. Ainsi, le Chili a créé en janvier un fond de stabilisation du pétrole, financé par les revenus du cuivre, qui s'élèvent à environ 200 millions de dollars ; le Salvador a créé un fond identique. Les pays exportateurs de pétrole, comme le Venezuela et l'Équateur, ont financé des fonds de réserve avec les rentrées supplémentaires de pétrole, et le Mexique a créé un fond de contingentement, avec comme objectif de prévenir d'éventuelles chutes des prix pétroliers.

Mais on devait rapidement se rendre compte que ces mesures et, tout particulièrement, la décision vénézuélienne d'augmenter la production, pour se donner les moyens de tenir la promesse faite aux Etats-Unis de leur fournir le pétrole nécessaire à leur effort militaire, n'ont pas moins agité l'opinion latino-américaine qu'une certaine perception de la nature du conflit du Golfe, de ces causes économiques, politiques, historiques et 
éthiques. A quelques niveaux privilégiés de l'expression politique, nous allons tenter d'analyser les différentes réactions de la société vénézuélienne et suivre leurs évolutions au long des différentes étapes chronologiques du conflit: la crise diplomatique, du 2 août au 16 janvier; la guerre aérienne, du 17 janvier jusqu'au 21 février, début de l'attaque terrestre; et enfin, du 21 février jusqu'au cessez-le-feu, l'effondrement irakien.

L'attitude des milieux universitaires et des intellectuels à l'égard du conflit

Dans le secteur étudiant et chez les universitaires, on a en général enregistré des réactions de soutien à l'Irak, qui s'inscrivent dans le prolongement d'une "posture antiimpérialiste" traditionnelle et fondamentalement anti-américaine. De ce fait, il n'y a pas eu de manifestations massives de soutien à l'Irak ou de condamnation de l'action des alliés, comme ce fut le cas lors de la guerre du Vietnam ou de l'invasion américaine à Panama. On peut néanmoins observer que des intellectuels ont multiplié des marches pacifistes et des protestations, tandis que divers débats étaient organisés dans les universités.

Dans Hora Universitaria, journal de la plus importante université du pays (l'UCV Université centrale vénézuélienne), le recteur Luis Fuenmayor Toro publia un article intitulé "Honte à la position vénézuélienne face à l'agression subie par l'Irak", qui peut être considéré comme représentatif de l'opinion de ce secteur. L'auteur rappelle que le Koweït fut une invention de l'Angleterre, dans le cadre d'une politique, menée conjointement avec la France, de création de régimes monarchiques pour diviser "la grande nation arabe"... "30 ans après, le protectorat britannique au Koweït, est devenu indépendant, comme s'il était une vraie nation (sic). Cette situation a toujours été contestée par l'Irak qui revendique le territoire koweïtien comme étant le sien. L'Angleterre a eu une attitude similaire en dépouillant par la force le Venezuela de la Guyane". Plus loin, le recteur signale à propos de l'invasion irakienne au Koweït... "On parle cyniquement de libérer le Koweït mais il est clair pour tout le monde que dans la soi-disant invasion irakienne on ne trouve aucun mort puisqu'il n'y eut aucune résistance. Tout s'est réduit à la destitution d'un gouvernement de marionnettes, saboteurs de l'OPEP, défenseurs des intérêts des grandes puissances (...) En réalité, l'armée irakienne a libéré le Koweït ${ }^{3}$. Les actions pacifistes que nous signalions plus haut, non seulement condamnent l'intervention américaine et alliée, mais critiquent durement la position du gouvernement vénézuélien dans le conflit et particulièrement sa politique pétrolière, comme le montre le slogan "Pas une goutte de pétrole vénézuélien pour la guerre du Golfe".

D'autres intellectuels ont adopté des positions qui ont varié au cours des différentes phases du conflit. En premier lieu, ils ont condamné la brutalité irakienne de l'annexion du Koweït, en l'accusant d'avoir violé le droit international. Ils ont exprimé une certaine méfiance à l'égard de la prétention de Saddam Hussein à devenir leader du tiers-monde alors que pendant la guerre Irak-Iran il avait fait alliance avec l'Occident. Ils ont pareillement condamné le type de gouvernement autocratique existant en Irak, les tueries et les répressions contre la population kurde ou les courants d'opposition. Mais les mêmes n'ont pas manqué de condamner "l'ingérence et la politique belliciste" des USA et de ses alliés en les accusant de mener une politique dont l'objectif essentiel était de contrôler les exportations de pétrole dans des conditions favorables aux Occidentaux. De fait, l'action des Américains ne s'exerce-t-elle pas au service de leurs propres intérêts, dans le but de leur assurer une position hégémonique dans le monde 
capitaliste ? Le temps n'est plus où l'Union soviétique, aujourd'hui économiquement et politiquement affaiblie, toute préoccupée de sa propre restructuration soutenait la cause du tiers-monde. Ce ne serait donc pas par hasard qu'elle a obtenu de l'Arabie Saoudite un crédit de 7 milliards de dollars pour prix de son soutien politique aux coalisés.

Les intellectuels vénézuéliens ont interprété le conflit du Golfe comme étant avant tout une manifestation claire de la confrontation Nord/Sud. L'Irak est un pays du tiersmonde qui a mené une politique nationaliste, et s'est engagé dans un projet de développement progressiste édifiant un régime "socialiste arabe". L'Irak a envahi et annexé le Koweït dans le but de mettre fin à "l'infâme régime autoritaire et monarchique" des Al Sabah au Koweït, dont la classe dirigeante a dilapidé les ressources provenant du pétrole, ce qui est, selon eux, un véritable scandale pour le reste du monde arabe dont les peuples pâtissent gravement de la distribution inégale de la richesse dans la région. Ces intellectuels contestent jusqu'au droit du Koweït à la souveraineté et soutienne la thèse des droits historiques de l'Irak sur le Koweït, bien qu'ils le fassent sans beaucoup d'arguments, révélant ainsi une grande ignorance de l'histoire du Golfe et du monde arabe en général.

Pour eux, peu importe le caractère anti-démocratique du régime irakien ou le soutien qu'il obtint de l'Occident pendant la guerre avec l'Iran. Dans ce dernier cas, il s'agissait avant tout d'une manoeuvre tactique destinée à contenir l'islamisme iranien. A ce moment-là, l'Occident avait accordé à l'Irak un soutien militaire et financier sans beaucoup s'émouvoir de la nature anti-démocratique et expansionniste du régime, apposant, pour ainsi dire, à la politique de Saddam Hussein, le sceau de la légitimité, comme l'atteste la complicité occidentale dont il a bénéficié pour s'armer. I1 n'est donc pas surprenant qu'il ait utilisé les armes et la technologie de l'Occident pour conduire une politique d'unification du monde arabe légitime pour beaucoup, et résoudre, par la manière forte, des problèmes endémiques de la région, largement négligés par la diplomatie internationale: les problèmes palestinien et libanais, par exemple. L'invasion du Koweït serait alors, si l'on suivait ce raisonnement, à la fois un acte de légitime justice sociale - pour mettre fin à l'excessive concentration des richesses par une tentative de restitution à l'Irak d'une province dont elle a été dépouillée par le colonialisme; et en même temps un appel désespéré à tous pour permettre une solution rapide des problèmes de la région.

Ce type d'analyse repose sur la grande confiance qu'avaient les intellectuels vénézuéliens dans la capacité militaire de l'armée irakienne. Ils espéraient que la prétendue "quatrième armée du monde" serait capable sinon d'infliger une sérieuse défaite aux armées américaines et alliées, du moins de transformer le conflit en un nouveau Vietnam pour les forces américaines. En ce qui concerne l'autocratisme du régime irakien, on n'en faisait peu de cas, soulignant qu'aucun régime au Moyen-Orient ne pouvait être considéré comme démocratique. De cette façon, on arrivait à la conclusion que les masses arabes étaient condamnées irrémédiablement à vivre sous des régimes de terreur. Le soutien politique à l'invasion irakienne est également justifié par la comparaison avec celui, considéré comme absolument légitime, que toute l'Amérique latine avait accordé à l'Argentine pendant la guerre des Malouines; or cette guerre avait été provoquée par un geste désespéré d'une dictature militaire argentine aux abois et condamnée par tous les peuples de cette région. Par analogie, il s'agissait à propos de l'Irak, d'appuyer une fois encore l'action d'un pays du tiers monde, 
s'opposant aux Etats-Unis, et à la coalition des pays industrialisés. On espérait que cette action pourrait ouvrir une brèche dans les rapports de domination qui structurent les relations internationales et qu'éventuellement cela pourrait se traduire par une amélioration de la position du tiers monde face au Nord.

La position des leaders et des partis politiques

Les trois partis politiques qui dominent la vie politique vénézuélienne sont: Accion Democratica (AD), COPEI et le Movimiento al Socialismo (MAS). Ils se considèrent comme les représentants de trois courants idéologiques: la démocratie sociale le christianisme social et le socialisme. Les deux premiers partis, qui alternent depuis trente ans au pouvoir, n'ont pas pris de position officielle face au conflit, à la différence du mouvement socialiste dont l'attitude a été fixée dans un communiqué.

Dans le cas des deux partis majoritaires (AD et COPEI), ce silence délibéré s'explique, à notre avis, par le contact établi avec la communauté d'origine arabe, qui compte plus de 200000 personnes. Des associations communautaires au Venezuela se sont manifestées à travers un soutien politique et financier, particulièrement pendant les périodes électorales. Cependant, le poids de cette communauté en ce qui concerne la définition de la politique extérieure, par les partis politiques et le gouvernement, ne peut être comparé en aucune manière avec la position influente de la communauté juive dans le monde politique vénézuélien. Son poids se marque par le refus réitéré des gouvernements vénézuéliens successifs, à autoriser l'ouverture d'un bureau de l'OLP à Caracas. Le Venezuela se distingue ainsi de la position des autres pays latinoaméricains, et ce, bien que sa politique officielle, au moins théoriquement, reconnaisse le droit à l'auto-détermination des Palestiniens, choix confirmé pendant le conflit du Golfe.

Toutefois, les dirigeants de chacun de ces partis ont exprimé leur position personnelle au Congrès national et à travers les médias. En terme général, le monde politique pense que, si l'invasion irakienne est condamnable, l'intervention des Etats-Unis et de ses alliés l'est aussi, puisque elle tente d'imposer l'hégémonie américaine dans une région cruciale sur le plan stratégique. En même temps, le destin de l'OPEP, et l'avenir du pétrole vénézuélien inquiètent: si le conflit du Golfe aboutit à une victoire alliée écrasante, l'emprise américaine sur le marché pétrolier serait alors étouffante puisque les Etats-Unis deviendraient en quelque sorte le quatorzième membre de l'OPEP.

Ceci a donné lieu à une polémique entre experts pétroliers, de diverses tendances politiques, sur le maintien ou la suppression de l'OPEP et sur la participation du Venezuela à cette organisation. Cette polémique est devenue plus vive après la victoire alliée, puisque les craintes de mise sous tutelle ont été justifiées par une déclaration d'un haut fonctionnaire koweïtien, qui envisageait favorablement une intervention directe américaine et alliée dans la fixation des prix du pétrole et du niveau de la production comme compensation à la participation de ces pays à la "défense du pétrole" et de la "stabilité régionale". A cet égard, la position de Caracas est de penser que le pays a intérêt à rester au sein de l'OPEP Cette position est maintenant partagée par des secteurs anciennement opposés au maintien du Venezuela dans l'organisation, puisqu'ils ont le sentiment qu'il n'existe aucune autre alternative.

30 La position du MAS, définie dans le communiqué publié dans la presse ${ }^{4}$, condamne l'invasion de l'Irak au Koweït mais accuse aussi les Etats-Unis de pousser à la logique de guerre sans tenir compte des tentatives de solutions pacifiques des autres pays, y 
compris de ses alliés et critique la position de l'ONU pour ne pas avoir déployé suffisamment d'énergie pour aboutir à une solution politique.

Des organisations politiques de gauche, dont certains dirigeants ou des militants ont été directement ou indirectement impliqués dans le mouvement guérillero vénézuélien des années 1960, ont dans leur grande majorité, apporté leur soutien à Saddam Hussein pendant le conflit. Ils ont reconnu en lui un nouveau et important leader du tiersmonde. Ce soutien, qui a quelquefois atteint le "niveau de l'euphorie", s'est manifesté pendant la guerre, tout au moins avant la bataille terrestre marquée par la lamentable prestation de l'armée irakienne. A cet égard, il paraît utile de donner l'opinion de Domingo Alberto Rangel, important leader politique et idéologue du "Movimiento de Izquierda Revolucionaria" (MIR), car elle reflète bien la pensée d'une gauche non liée aux partis politiques: (...) "En occupant le Koweït ou en y étant présentes, les forces militaires irakiennes et leur chef Saddam Hussein ont donné la preuve que la révolution n'était pas morte ni atteinte d'une maladie gui nécessiterait une thérapie intensive (...) Bien que nous n'ayons rien à voir avec Saddam Hussein du point de vue idéologique, nous autres révolutionnaires du monde avons contracté une grande dette avec lui (...) Saddam Hussein figurera parmi les grands bienfaiteurs de l'humanité opprimée" 5 .

L'opinion publique, les médias et le conflit

L'opinion publique vénézuélienne, assez influencée par les médias a changé d'orientation au cours des différents moments du conflit. Fin octobre, par exemple, c'est à dire avant la guerre, un sondage d'opinion a été réalisé avec un échantillon de 863 personnes à Caracas et à Maracaïbo. Il donne les résultats suivants :

- $28 \%$ ont considéré que l'Irak avait envahi le Koweït pour raison économique, 23,3\% parce que le Koweït était une province irakienne, et 29,2\% pour raison religieuse ; 4,3\% pour ne pas payer la dette extérieure ; $6,1 \%$ pour ne pas maintenir les prix du pétrole à la baisse, $6,2 \%$ pour raisons politiques.

- Pour 35,5\%, les Etats-Unis ont envoyé des troupes dans le Golfe "pour défendre le pétrole bon marché", 20,6 \% "pour freiner le pouvoir de Saddam Hussein", 14,1 \% pour garantir le respect des frontières".

- Quelles sont les responsabilités de Saddam Hussein et de George Bush dans le conflit? Pour 41,7 \% des Vénézuéliens, le responsable était Saddam Hussein, pour 17,2 \% c'était Bush, tandis que $22,6 \%$ les accusaient tous les deux.

- A la question : lequel de ces deux leaders avaient raison, $36,8 \%$ les renvoyaient dos à dos, $25,2 \%$ étaient pour Bush et $24,5 \%$ pour Saddam Hussein.

- Quant à l'impact de la crise sur le Venezuela, $27 ; 6 \%$ ont considéré que la position du gouvernement était bonne, 19,7 \% considérait qu'elle n'était pas bonne, 23,9\% jugeaient que le gouvernement n'assumait aucune position. A propos des conséquences du conflit sur le pays et du sort des revenus pétroliers additionnels, 65,6 \% ont considéré que la crise bénéficiait au Venezuela et $33,8 \%$ ont jugé que l'escalade des prix était préjudiciable au pays. Dans le premier groupe, $57,4 \%$ ont cru que le Venezuela aurait plus d'argent pour surmonter sa propre crise et $21,3 \%$ qu'il pourrait ainsi 
rembourser sa dette extérieure. Quant aux autres, 50,9\% ont considéré que le Venezuela gaspillerait plus d'argent dans la corruption et dans la bureaucratie, 12,7 \% ont exprimé la crainte que les pays industrialisés remplacent le pétrole par d'autres énergies et 9,1\% ont craint qu'une crise des pays industrialisés puisse les obliger à diminuer leurs achats de pétrole.

38

- Quant à la gestion de ces ressources par le gouvernement actuel, seuls $19 \%$ des sondés pensaient qu'elles seraient bien gérées $39 \%$ que cette gestion serait passable et $35 \%$ qu'elle serait mauvaise ${ }^{6}$. parallèles à la chronologie réelle du conflit. Durant la pré-guerre, les médias ont donné aux événements une couverture ample à partir d'informations provenant des agences internationales, des réseaux d'information par satellite complétées par l'intervention de journalistes, d'ingénieurs $d u$ secteur pétrolier d'hommes politiques et d'internationalistes, "subitement transformés en experts sur le Moyen-Orient".

Parallèlement, il y a eu un vaste débat sur les perspectives du marché pétrolier international, sur la hausse des prix, qui a fini par avaliser le pronostic de l'ancien ministre saoudien Zaki Yamani qui avait pronostiqué que le prix du baril atteindrait de 60 à 100 dollars. On a également discuté des conséquences sur l'économie des ressources supplémentaires, de leur emploi.

41 Le premier jour de la bataille aérienne, la retransmission permanente des images de guerre et des performances "opérationnelles" des alliés par les médias audiovisuels, reliés à des chaînes en majorité américaines, comme CBS et CNN, a effrayé la population: celle-ci s'est précipitée dans les magasins, et a commencé à stocker des vivres de façon irrationnelle dans la crainte que la guerre pourrait provoquer la diminution des importations et une pénurie d'aliments et d'articles de consommation.

Le gouvernement s'est inquiété de ces réactions. Il craignait qu'une situation de pénurie puisse s'ajouter aux effets sociaux du programme d'ajustement structurel et à ceux de la spéculation, puis reproduise un ensemble de conditions similaires à celles qui avaient existé juste avant l'explosion sociale du tristement célèbre 27 février $1989^{7}$. Le gouvernement a alors imposé - non officiellement - une censure aux médias. Ceux-ci étaient d'ailleurs accusés par d'autres secteurs de l'opinion, comme les associations civiles ou les associations de quartiers de traiter l'information d'une façon d'autant plus alarmiste, qu'il y avait entre les chaînes de télévision une lutte acharnée pour le "rating". Cette situation a provoqué d'importants effets psychologiques chez les jeunes téléspectateurs, créant une sorte de "psychose de guerre". Le changement de la politique des chaînes de télévisions a été marqué par un rééquilibrage entre l'information locale, ou nationale, et l'information sur le Golfe rééquilibrage facilité par la diminution de la quantité des informations en provenance du Golfe, provoqué cette fois-ci par la censure des pays directement dans le conflit.

43 Dans la deuxième et troisième étape de la guerre du Golfe, les positions ont été moins divergentes que dans la première. A l'approche de l'expiration du délai accordé à l'Irak à l'ONU pour l'évacuation du Koweït, l'incertitude sur le dénouement du conflit se mélangeait avec l'espoir existant dans l'opinion publique, les médias, les intellectuels, étudiants et professeurs, que Saddam Hussein et l'armée irakienne seraient capables de causer des pertes importantes à l'armée américaine, ou d'obtenir une victoire au moins politique comme ce fut le cas pour le Vietnam. On saisissait là le désir de voir les Etats- 
Unis punis de tout ce qu'ils pouvaient faire impunément sur le continent américain, notamment tout récemment, à la Grenade et au Panama.

La faible résistance de l'armée irakienne à l'écrasante attaque aérienne a provoqué un sentiment général de désillusion. Mais dans les secteurs les plus favorables au leader irakien, cette passivité était interprétée comme une simple apparence recouvrant un brillant stratagème militaire qui pourrait surprendre le commandement américain et les alliés par un coup inattendu, en opposantà la puissance technologique la ruse, considérée comme le grand recours du tiers-monde. Cette perception s'est trouvée renforcée quand les missiles Scud, que l'on avait cru détruits, ont été lancés sur Israël et l'Arabie Saoudite depuis les plates-formes mobiles savamment dissimulées; on se plaisait à croire que les Américains n'avaient détruits que le matériel de camouflage de ces mêmes missiles, utilisé comme leurre par l'armée irakienne.

Cependant les missiles Scud lancés sur Israël, dans une vaine tentative pour impliquer directement ce pays dans le conflit, ont été l'occasion de la publication d'un communiqué, signé par des intellectuels et artistes vénézuéliens de gauche, qui a surpris beaucoup de gens. Ce communiqué condamnait les attaques contre le peuple israélien. Quelques associations arabes ont répliqué que ces attaques étaient justifiées par des menées - passées et présentes de l'appareil militaire et répressif israélien contre le peuple palestinien dans les territoires occupés et d'une manière générale par la politique appliquée contre les autres Arabes. Ils ont critiqué l'absence de sensibilité de ces intellectuels et de ces artistes face à la souffrance du peuple palestinien.

$\mathrm{Au}$ fur et à mesure que les effets de la campagne aérienne paraissaient de plus en plus catastrophiques pour l'Irak, la condamnation des alliés, accusés de détruire sans pitié un petit pays et son peuple, prit de l'ampleur. Cette condamnation est devenue plus énergique après la mort de 500 Irakiens pendant le bombardement américain d'un abri anti-aérien. En même temps, la frustration des observateurs devant l'immobilité obstinée de l'armée irakienne, face à ce que l'on considérait comme le massacre de son peuple, augmentait. Mais l'espoir persistait que la situation pourrait changer avec la bataille terrestre; l'expérience acquise par l'Irak dans la guerre avec l'Iran, la connaissance du terrain et une certaine supériorité numérique contribueraient pensait-on à une victoire irakienne.

Dans la troisième étape, celle de l'attaque terrestre, marquée par la défaite écrasante de l'Irak sans que ce pays n'ait montré sa prétendue capacité militaire, le prestige de Saddam Hussein, comme leader du tiers monde, s'est détérioré vertigineusement. On ne le considérait plus comme l'homme qui aurait pu détruire le mythe de l'invincibilité des États-Unis et de ses alliés et venger ainsi toutes les injustices dérivées du système mondial de domination du Sud par le Nord.

48 A la désillusion politique provoquée par la passivité de Saddam Hussein et de son armée, s'ajoute celle entraînée par la politique pétrolière appliquée par les Etats-Unis et leurs alliés, dont les conséquences sont clairement apparues dans le comportement du marché pendant les deux dernières étapes du conflit. Au lieu d'un enchérissement, on a enregistré une baisse rapide et continue des prix du pétrole, ce qui a entraîné la crainte que le montant du baril passe au dessous de la barre des 10 dollars, comme c'était arrivé lors du contre-choc pétrolier des années 80 . Cette baisse des prix a été, estime-t-on, la conséquence de la politique menée par les Etats-Unis puisque ce pays a mis sur le marché ses réserves stratégiques, empêchant la montée des prix et les contraignant à la baisse. De tels agissements, ainsi que la politique pétrolière 
vénézuélienne au cours de la crise, ont été sévèrement critiqués par les partis politiques et par les experts pétroliers, soutenus en cela par des secteurs importants de l'opinion publique. Ces critiques se sont exprimées dans les déclarations de hauts dirigeants de deux grands partis politiques : AD et COPEI. M. Umberto Celli, secrétaire général d'Action démocratique, parti au pouvoir, a signalé que "les responsables du comportement du marché pétrolier sont les puissances industrialisées qui ont trompé les bonnes intentions des pays, qui comme le Venezuela, ont agi de bonne foi en augmentant leur production pour remplir le vide laissé par l'Irak et le Koweït"8.

Le secrétaire général adjoint du COPEI a considéré, quant à lui, que "le gouvernement a pratiqué une politique pétrolière immédiate et myope"

La presse nationale s'est montrée sensible à ces interprétations et à ces critiques, considérant l'intervention américaine sur le marché pétrolier comme un stratagème supplémentaire prouvant une fois de plus la nature égoïste de la politique des pays industrialisés à l'égard de pays, qui comme le Venezuela, dépendent de la production de matières premières. La presse a étendu en même temps ses critiques contre le gouvernement, le qualifiant de "complaisant" et "sans capacité de négociation".

51 L'image de Saddam Hussein comme leader tiers-mondiste, après avoir été au zénith pendant la crise, s'effondre pendant la guerre. Cette évolution est confirmée par la période d'après-guerre où la répression barbare par Saddam Hussein des soulèvements kurdes au Nord et chi'ites au Sud, qui contrastait avec la faible réponse irakienne aux armées occidentales, choque l'opinion vénézuélienne.

La vision politique du conflit dans le reste de l'Amérique latine

52 Dans le reste de l'Amérique latine, la perception du conflit du Golfe et les prises de position ont été très similaires à celles enregistrées au Venezuela. La plupart des gouvernements latino-américains ont soutenu les résolutions adoptées par l'ONU. Ces pays ont condamné l'invasion irakienne au Koweït mais se sont abstenus d'approuver l'utilisation de la force pour la repousser. On a cependant remarqué des positions divergentes.

53 Le gouvernement argentin a été dans toute la région, le seul à envoyer un contingent militaire avec deux bâtiments de guerre, "le Spriro" et "l'Almirante Brown", en déclarant que le but de ce contingent n'était que de donner un soutien logistique. La position adoptée par le président Menem a été l'objet d'attaques au Congrès de son pays par l'opposition et notamment par l'Union civile radicale, dirigée par l'exprésident Alfonsin qui exigeait le retour immédiat des bâtiments envoyés dans le Golfe, en signalant que cette décision n'avait pas été autorisée par le Congrès. L'opinion publique argentine ou latino-américaine en général a sévèrement critiqué les quelques déclarations $\mathrm{du}$ Président, les considérant comme "extrêmement légères" et condamnables. Le président Menem pensait que les répercussions économiques de la guerre seraient bonnes pour les Argentins puisque le pays pourrait profiter de l'augmentation de la demande mondiale d'aliments et de combustibles 9 . Dans toute l'Amérique latine se sont déroulées des manifestations pacifistes et anti-américaines avec de nombreuses marques de sympathie à l'égard de Saddam Hussein. En Colombie, par exemple, selon un sondage réalisé par le journal Santa Fé de Bogota dans un échantillon de 1000 personnes, 419 étaient favorable à Saddam Hussein, 379 aux EtatsUnis et 206 neutres. Le commentaire soulignait qu'un grand pourcentage de la population a condamné l'attitude des Etats-Unis, sans soutenir Saddam Hussein, mais néanmoins sans lui reprocher d'avoir envahi le Koweït. Ceux qui se sont montrés 
opposés à la politique des Etats-Unis avançaient l'argument selon lequel Washington, après avoir envahi la Grenade et Panama ${ }^{10}$, n'avait pas l'autorité morale requise pour intervenir dans le Golfe persique. On peut expliquer le soutien que Saddam Hussein a trouvé dans de nombreux secteurs du continent latino-américain - surtout avant sa défaite - par son image de leader millénariste. Le dirigeant irakien en effet était considéré comme un "sauveur" et même comme un "vengeur", qui émergeait à travers un discours apocalyptique, dans un moment particulièrement inquiétant de "fin du monde". Contre un certain ordre mondial qui entraîne dans la région de grandes tensions sociales et économiques, il avait essayé de mettre fin à une "décennie perdue", face à la grande incertitude de l'avenir.

Comme on a pu le souligner dans cet article, la vision vénézuélienne et latinoaméricaine de la crise du Golfe arabo-persique, a été influencée par les multiples contradictions existant entre le monde latino-américain et les Etats-Unis, contradictions qui sont le résultat d'événements anciens et nouveaux, mais qui ont laissé des ressentiments profonds. Dans ce sens, on peut dire que cette vision est la projection de "l'image de soi" des latino-américains qui se sentent les victimes des Etats-Unis en particulier ou des pays industrialisés en général. Cette vision est aussi la conséquence de la méfiance de la région pour le rôle qui serait attribué à l'Amérique latine dans le nouvel ordre mondial, préparé par l'administration Bush, reposant sur des Etats-Unis triomphants, et sur l'écroulement du monde socialiste d'autrefois.

\section{NOTES}

1. OPEC Secretariat Energy and Oil statitic, Vienne, décembre 1990, pp. 101-110.

2. OLADE, CEPAL, SELA, Amérique latine et Caraibes : Représentations de la crise du Golfe et options pour y faire face, carta Semanal, Caracas, Ministère de l'énergie et des mines, $n^{\circ} 1640,2 / 1991$.

3. La Hora Universitaria, Caracas, 10ème année, $n^{\circ} 59,2 / 1991$, p. 2

4. El Universal, Caracas, 10/01/1991.

5. Domingo Alberta Rangel, debemos un monumento a Saddam Hussein, El mundo, Caracas, 11/1991.

6. Enquête réalisée par l'entreprise Multisigno, Encuesta sobre el Golfo Pérsico : los Venezolanos opinan con madurez, Revue Zeta, n 827, 11/1991, pp. 22-25.

7. Début des manifestations émeutières qui ensanglantèrent Caracas du 27/02 au 7/03 1989.

8. Journal Economia Hoy, Caracas, 1/1991, p. 4.

9. Journal Economia Hoy, Caracas, 1/1991, p. 23.

10. Référencé par l'AFP, reproduit dans El Nacional, Caracas, 1/1991, p. A-2. 


\section{RÉSUMÉS}

Proche des Etats-Unis, quoiqu'un membre important de l'OPEP, le Vénézuela a été particulièrement affecté par la crise du Golfe. Le problème soulevé a porté sur le pétrole et l'utilisation pouvant être faite des gains supplémentaires liés au prix du baril, sur l'ampleur corrélative de la corruption, ainsi que sur la politique égoïste de Washington qui n'hésita pas à vendre ses réserves stratégiques sur le marché international. Par ailleurs, Saddam Hussein a trouvé un soutien parmi les Vénézueliens, cette attitude ayant été dictée par le sentiment antiimpérialiste encore dominant en Amérique Latine.

Close to the USA, but nevertheless an important member of OPEC, Venezuela was particularly affected by the Gulf crisis. Discussions concerned petrol and the use to be made of additional profits gained through the rising price of the barrel, the correlative amplitude of corruption and the selfishness of Washington's policy, not hesitating to sell its strategic reserves on the international market. At the same time, Saddam Hussein found supporters among Venezuelan people, an attitude dictated by the anti-imperialist feeling still prevalent in Latin America.

INDEX

Index géographique : Amérique du Sud

Mots-clés : conflits internationaux, guerre

Index chronologique : 1991

\section{AUTEUR}

\section{EVELYN BRAVO DIAZ}

CENTROPEP,Université Santa Maria à Caracas 